\title{
Locating seed points for automatic multi-organ segmentation using non-rigid registration and organ annotations
}

\author{
Ranveer Joyseeree ${ }^{a, b}$, Henning Müller ${ }^{a, c}$ \\ ${ }^{a}$ University of Applied Sciences Western Switzerland (HES-SO), Sierre, Switzerland; \\ ${ }^{b}$ Eidgenössische Technische Hochschule (ETH), Zürich, Switzerland; \\ ${ }^{c}$ Medical Informatics, University Hospitals \& University of Geneva, Switzerland;
}

\begin{abstract}
Organ segmentation is helpful for decision-support in diagnostic medicine. Region-growing segmentation algorithms are popular but usually require that clinicians place seed points in structures manually. A method to automatically calculate the seed points for segmenting organs in three-dimensional (3D), non-annotated Computed Tomography (CT) and Magnetic Resonance (MR) volumes from the VISCERAL dataset is presented in this paper. It precludes the need for manual placement of seeds, thereby saving time. It also has the advantage of being a simple yet effective means of finding reliable seed points for segmentation.

Affine registration followed by B-spline registration are used to align expert annotations of each organ of interest in order to build a probability map for their respective location in a chosen reference frame. The centroid of each is determined. The same registration framework as above is used to warp the calculated centroids onto the volumes to be segmented. Existing segmentation algorithms may then be applied with the mapped centroids as seed points and the warped probability maps as an aid to the stopping criteria for segmentation.

The above method was tested on contrast-enhanced, thorax-abdomen CT images to see if calculated centroids lay within target organs, which would equate to successful segmentation if an effective segmentation algorithm were used. Promising results were obtained and are presented in this paper. The causes for observed failures were identified and countermeasures were proposed in order to achieve even better results in the next stage of development that will involve a wider variety of MR and CT images.
\end{abstract}

Keywords: Medical image processing, 3D probability maps, automatic seed point detection, segmentation

\section{INTRODUCTION}

Clinicians have come to rely very heavily on imaging for diagnosis and pre-operative surgical planning. ${ }^{1}$ There is a clear need for the efficient processing of the information amassed. ${ }^{2}$ Segmentation is one example of such processing and, for region-growing segmentation, seed points need to be manually placed by experts. Time that could otherwise be used for better patient care is thus needlessly spent. An algorithm for the automatic identification of seed points for region-growing segmentation would preclude such a situation. After segmentation, further processing of organ tissue such as 3D texture analysis can then be performed ${ }^{3}$ or other decision support tools can use the input data. ${ }^{4}$

Several related approaches have been published in the literature. Singh et al. ${ }^{5}$ propose a method that calculates seed points from areas of interest in grayscale two-dimensional (2D) images based on background and object properties. This is followed by a region-growing stage using those seed points. Their method was, however, not implemented for 3D images and was demonstrated only for X-ray images.

Xing et al. ${ }^{6}$ use statistical fusion of 3D brain MR images to find seed points for segmentation. Their method incorporates the MAP-CSTAPLE ${ }^{789}$ approach to minimise mean-squared error(MSE). Our approach is simpler since we aim to verify only if calculated seed points fall within the target organ. They also use FNIRT ${ }^{10}$ for registration while we use the more powerful and general combination of affine and B-Spline registration within the elastix framework. ${ }^{11}$ Our method is, therefore, suitable for any organ of interest as long as expert annotations for them exist.

Further author information: (Send correspondence to Ranveer Joyseeree, Email: ranveer.joyseeree@hevs.ch) 
In addition, a hybrid region-growing algorithm was proposed by Mubarak et al. ${ }^{12}$ The technique finds seeds using the Harris corner detection. ${ }^{13}$ However, it was not extended to 3D images and was tested only on $\mathrm{X}$-ray images of the knee and 2D MRI brain images. Our method is comparatively more complicated but it is applicable to $3 \mathrm{D}$ volumes of any organ.

It is clear from the above that our method presents distinct advantages as compared to previous techniques developed in the field. Furthermore, this study is well aligned with the FP7-funded Visual Concept Extrac-

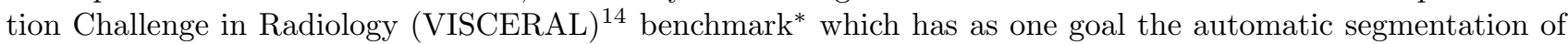
anatomical structures in non-annotated full-body MR and CT volumes. The project also involves the identification of an unspecified organ for which training data need to be analyzed without a-priori knowledge of the organ. A segmentation method that uses the proposed method of seed point identification would well-suited to achieve the goals of VISCERAL without the need to manually optimize and adapt tools to specific organs.

\section{METHODS}

A series of MR and CT volumes $Y_{1}$ to $Y_{N}$ (where $N$ is the size of the set of available volumes) is provided by VISCERAL. Each acquisition was examined by expert radiologists who annotated up to 20 organs and saved each annotation, labelled $\mathrm{A}\left(Y_{n}\right.$,organ) (where $\mathrm{n}$ is the identifier of a particular volume in the database), as a separate volume.

One element of the set $\left\{Y_{1}, Y_{2}, \ldots, Y_{N}\right\}$ is picked as the reference frame and labelled as $X_{i}$ (where $i$ goes from 1 to $N)$. It follows that: $X_{i} \in\left\{Y_{1}, Y_{2}, \ldots, Y_{N}\right\}$. This is used as a reference volume for the remaining steps in this section and the whole process is repeated several times with a different element picked from $\left\{Y_{1}, Y_{2}, \ldots, Y_{N}\right\}$ until all the members of the latter have been used as a reference once.

Figure 1 illustrates an instance of $X_{i}$ and includes an illustrative annotation, $A\left(X_{i}\right.$, liver $)$, which is displayed as a bright white region on top of $X_{i}$. The visualisation was generated using $3 \mathrm{D}$ Slicer ${ }^{\dagger 15}$

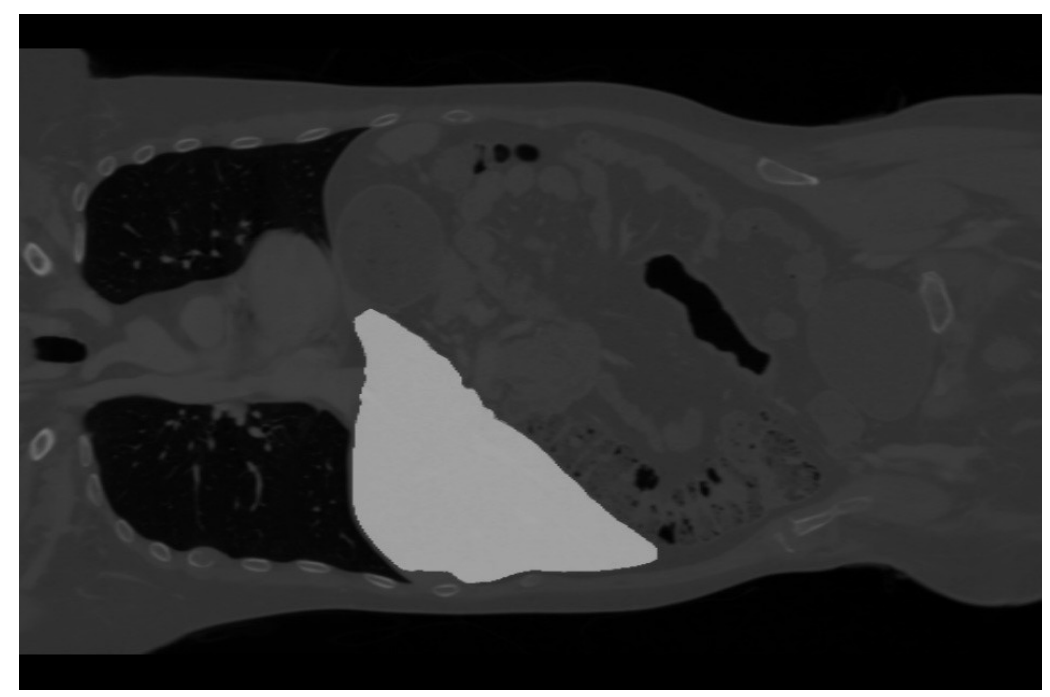

Figure 1: A contrast-enhanced, thorax-abdomen CT volume is chosen as $X_{i}$. For illustrative purposes, $X_{i}$ is superimposed with its corresponding liver annotation (manually generated by an expert): $A\left(X_{i}\right.$, liver), shown as a bright white region.

*VISCERAL benchmark: http://www.visceral.eu/closed-benchmarks/benchmark-1/, 2012. [Online; accessed 19January-2015].

${ }^{\dagger} 3$ D Slicer: http://www.slicer.org/, 2013. [Online; accessed 19-January-2015]. 


\subsection{Selection of volumes based on body shape}

The success of our method is highly dependent on the quality of registration between training volumes and the chosen reference volume. Minimising registration error is, thus, a top priority. Separating volumes according to the body shape of the patients involved and subsequently applying our algorithm on patients having similar body shapes separately would contribute greatly to reducing such errors. That is because, for instance, organ localisation would be wildly different for an underweight patient as compared to an obese one but, amongst underweight or obese patients separately, organ placement would be very similar. We, therefore, proceed to assess the body shape of patients via a close inspection of their medical scans.

\subsection{Registration}

A training volume from a certain class of body shape, labelled as $Y_{1}$, is then registered with $X_{i}$ belonging to the same class of body shape. $Y_{1}$ is chosen as the moving volume and $X_{i}$ is the fixed volume. An affine registration $^{16}$ followed by a $\mathrm{B}$-spline registration ${ }^{17-19}$ are carried out because this combination offers better accuracy as compared to only rigid or affine registration methods. The cost metric used is mutual information (MI). ${ }^{20}$ To speed up the computation of MI, the implementation by Mattes et al. ${ }^{21,22}$ is used. To minimize the interpolation errors that occur during registration while keeping computation time low, B-Spline interpolation is carried out. After the successful completion of registration, the B-spline transform $T$ that maps $Y_{1}$ onto $X_{i}$ is obtained. Next, one organ of interest, $Z$, is picked. The annotation volume, $\mathrm{A}\left(Y_{1}, Z\right)$, is then converted into a binary volume before being transformed using $T$, giving $A^{T}\left(Y_{1}, Z\right)$. The latter is resampled such that it has the exact volume and voxel dimensions as $A\left(X_{i}, Z\right)$, which itself has the same mensurations as $X_{i}$.

\subsection{Creation of the Probability Distribution Volume}

To create a probability distribution volume with $X_{i}$ as the reference, the above registration step is carried out for all $N--2$ available VISCERAL volumes apart from $X_{i}$ itself and a volume, $X_{\text {test }}$, which is kept aside for testing and evaluating the method. For each training volume $Y_{n}$, where $n$ goes from 1 to $N--2$, a different transformation $T_{n}$ and a different warped annotated volume $A^{T_{n}}\left(Y_{n}, Z\right)$ are obtained. Since $A^{T_{n}}\left(Y_{n}, Z\right)$ for all $n$ have the same volume and voxel sizes as $A\left(X_{i}, Z\right)$, they may be combined together voxel-wise and then normalized according to equation 1 to obtain the probability distribution volume for organ $Z$ : $P D_{Z}$. Please note that $A\left(X_{i}, Z\right)$ and $X_{\text {test }}$ are excluded from the above calculation in order to avoid bias.

$$
P D_{Z}=\frac{1}{N} \sum_{n=1}^{N} A^{T_{n}}\left(Y_{n}, Z\right)
$$

\subsection{Generation of a Seed Point}

The centroid of $P D_{Z}$, represented in row vector form as $\left[\begin{array}{lll}x_{c} & y_{c} & z_{c}\end{array}\right]$, corresponds to the weighted average location of a point that lies within $P D_{Z}$. For an $M \mathrm{x} N \mathrm{x} P$ volume, it can be found using equation ( 2), where $V(x, y, z)$ is the voxel value at coordinates $(x, y, z)$, which is represented as $\left[\begin{array}{lll}x & y & z\end{array}\right]$ in vector form.

For the volume, $X_{\text {test }}$, on which the seed point for segmentation has to be found, B-spline registration between $X_{i}$ and $X_{\text {test }}$ is carried out. This time, $X_{i}$ is used as the moving image while $X_{\text {test }}$ is the fixed volume. The obtained transformation is applied to the volume containing the centroid found above. The location of the warped centroid may now be used as a seed point, $S\left(X_{\text {test }}\right.$, organ $)$, for segmentation on volume $X_{\text {test }}$.

$$
\left[\begin{array}{lll}
x_{c} & y_{c} & z_{c}
\end{array}\right]=\frac{\sum_{x=1}^{M} \sum_{y=1}^{N} \sum_{z=1}^{P} V(x, y, z) *\left[\begin{array}{lll}
x & y & z
\end{array}\right]}{\sum_{x=1}^{M} \sum_{y=1}^{N} \sum_{z=1}^{P} V(x, y, z)}
$$

\subsection{Evaluation of Viability of the Generated Seed Points}

To determine if $S\left(X_{\text {test }}\right.$, organ $)$ is a viable seed point for segmentation, it is sufficient to determine if it lies on a non-zero part of $A\left(X_{\text {test }}\right.$, organ $)$. That follows from the reasonable assumption that an effective region-growing algorithm will succeed if is supplied with a seed lying in the target organ. 


\section{RESULTS}

Fifteen contrast-enhanced, thorax-abdomen CT volumes in the VISCERAL dataset were considered for use in order to generate the results presented in this section. Full-body CT and MR volumes were not taken into consideration in this paper as correct registration across modalities and between thorax-abdomen and full-body volumes is more difficult to achieve and more liable to lead to errors. Since a discussion of the merits of our chosen registration method is out of the scope of this paper, incorporating such data would only distract us from our goal of demonstrating the usefulness of the seed point generation algorithm as a whole. Future work will incorporate those images as well and more in-depth work will be carried out to ensure good registration for all image types.

After classifying the patient body shape through careful examination of the CT scans, we found 11 normalweighing, 1 underweight, 2 overweight, and 1 obese patients. The method described earlier was applied to those 11 volumes as there are not enough patients for the other body shapes. In turn, one was chosen from the 11 as the reference, $X_{i}$, while another was chosen as the test volume, $X_{\text {test }}$. The remaining 9 volumes were used to generate the organ probability maps. Seven organs were selected for segmentation: the liver, spleen, right lung, left lung, right kidney, left kidney, and urinary bladder Therefore, a total of 770 experiments were conducted.

Figure 2a shows an example of the computed probability distribution volume for the liver, $P D_{\text {liver }}$, in coronal view. The lightest region indicates a probability of one for a voxel to lie on the liver and the darkest region indicates a probability of zero. Figure $2 \mathrm{~b}$ illustrates the centroid of $P D_{\text {liver }}$ as a very dark point near the centre of the probability distribution.

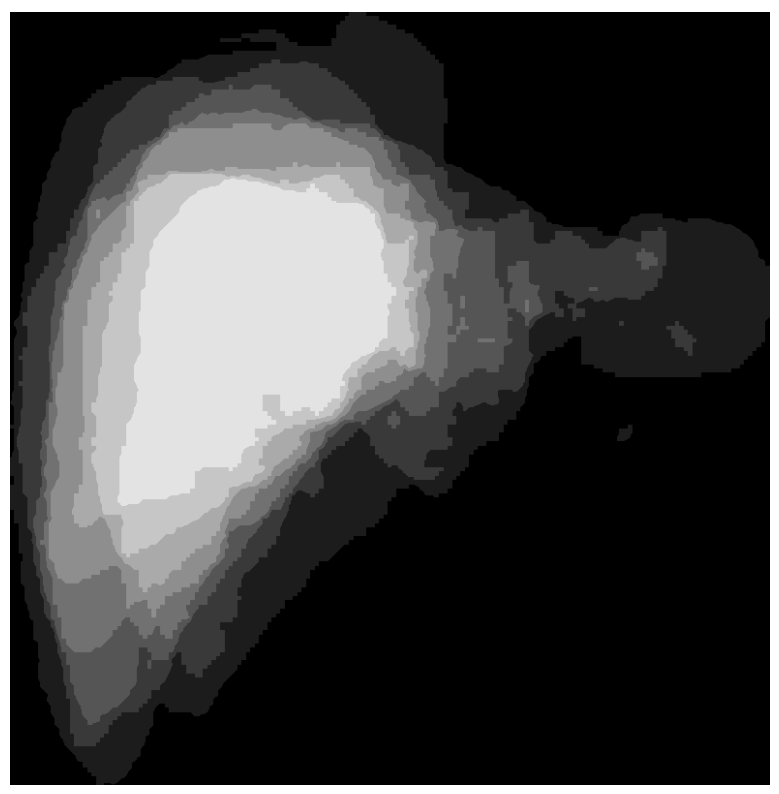

(a)

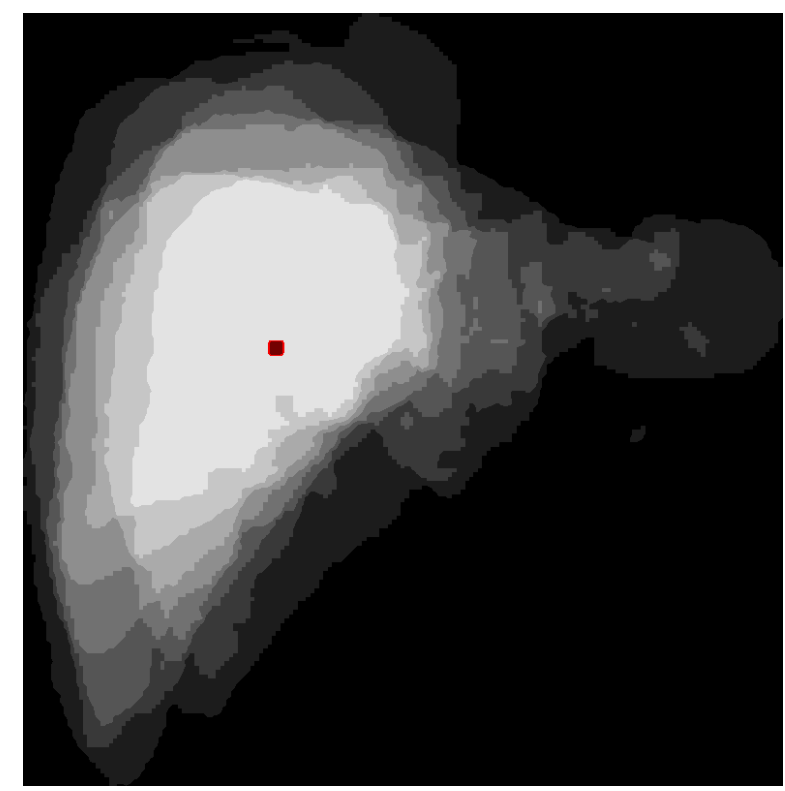

(b)

Figure 2: (a) The probability distribution volume of the liver (coronal view), produced using 9 liver annotations and superimposed on the reference volume is shown. The lightest region indicates a probability of 1 for a voxel to lie on the liver and the darkest region indicates a probability of 0; (b) Computed centroid of the liver shown as a dark spot in the middle of the probability distribution volume.

The result of applying the procedure separately on the liver, the right lung, the right kidney and the urinary bladder is shown in Figure 3. It may be observed that the seed points shown are located well within the target organs, implying that effective segmentation algorithms are expected to accurately segment those organs.

Table 1 shows how the volumes in the VISCERAL dataset are assigned new identification (ID) information to improve the simplicity and readability of Table 2 which shows the result of determining the viability of calculated 


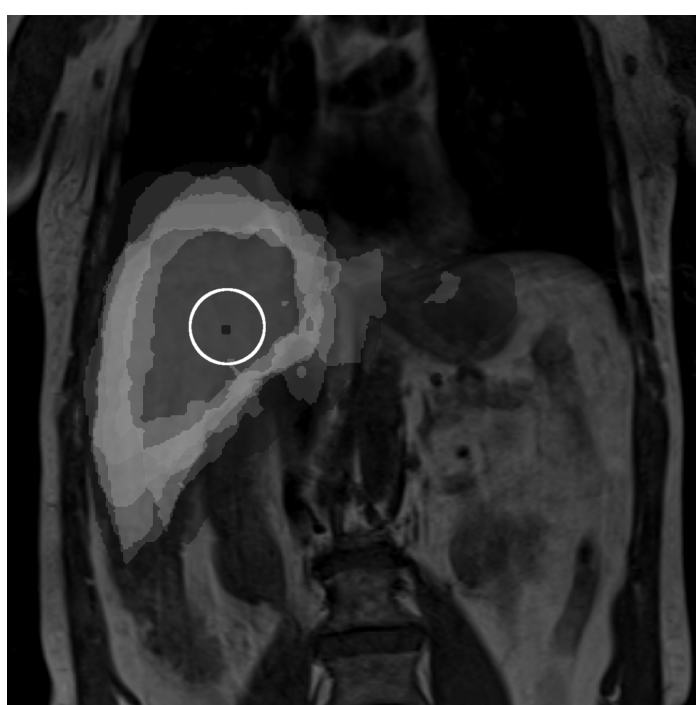

(a)

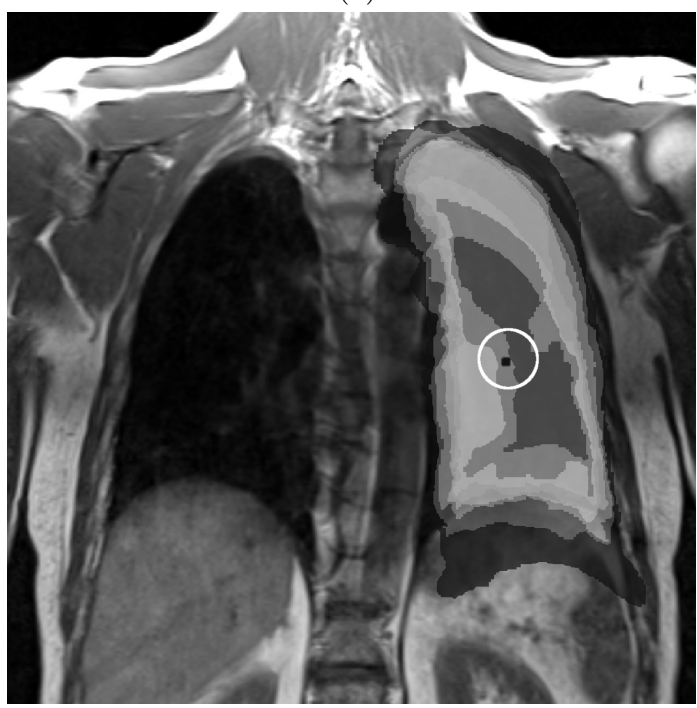

(c)

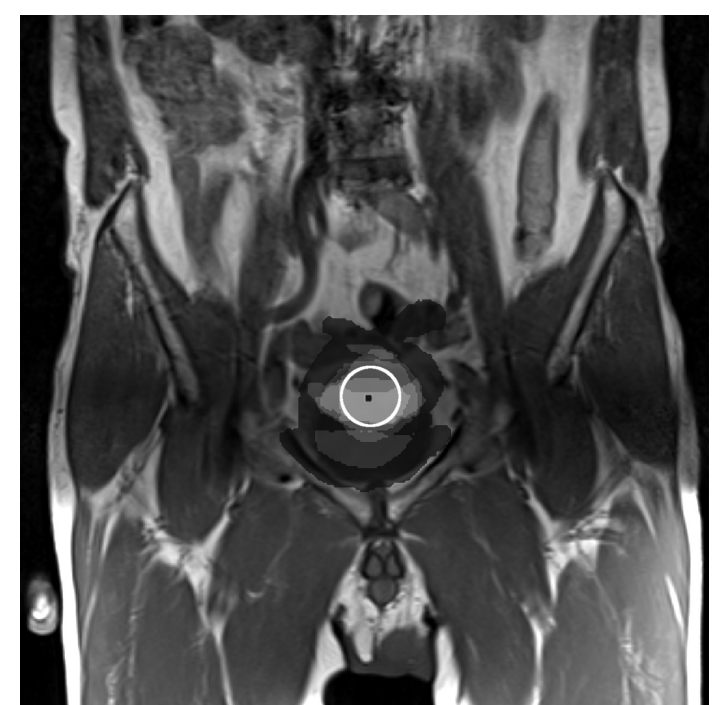

(b)

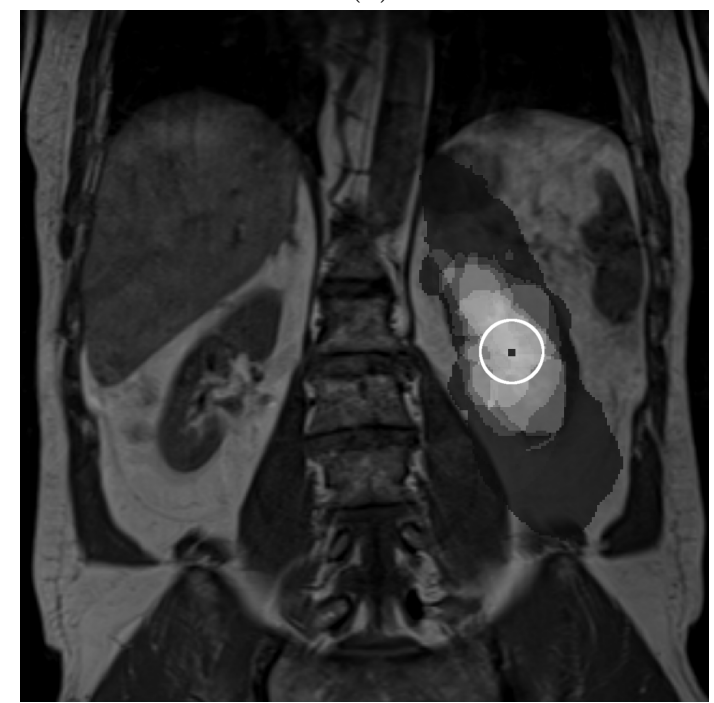

(d)

Figure 3: The above sub-figures show the following: (a) Encircled seed point superposed on $X_{i}$ and $P D_{\text {liver }}$; (b) Encircled seed point on $X_{i}$ and $P D_{\text {urinary_bladder }}$; (c) Encircled seed point superposed on X and $P D_{\text {left_lung }}$; (d) Encircled seed point on $X_{i}$ and $P D_{\text {left_kidney }}$. 
seed points for the 770 experiments. For each fixed volume and each organ of interest, 10 volumes were available for testing. Therefore, 10 seed points could be calculated per fixed volume and per organ. The ratio of the number of viable seed points (which can vary from 0 to 10) to the number of calculated seed points (which is 10 here) for a particular fixed volume and a particular organ of interest constitutes one entry in Table 2. Naturally, the ratio goes from 0.0 to 1.0 and there are 77 such entries corresponding to the 11 possible fixed images and 7 possible organs of interest.

Table 1: Images used from the VISCERAL dataset and the volume ID assigned to each in this paper.

\begin{tabular}{c|c} 
VISCERAL filename & Assigned Volume ID \\
\hline 10000100_1_CTce_ThAb & 100 \\
10000105_1_CTce_ThAb & 105 \\
10000106_1_CTce_ThAb & 106 \\
10000108_1_CTce_ThAb & 108 \\
10000109_1_CTce_ThAb & 109 \\
10000110_1_CTce_ThAb & 110 \\
10000112_1_CTce_ThAb & 112 \\
10000113_1_CTce_ThAb & 113 \\
10000127_1_CTce_ThAb & 127 \\
10000128_1_CTce_ThAb & 128 \\
10000131_1_CTce_ThAb & 131
\end{tabular}

Table 2: Results of conducting 770 experiments to find seed points for region-growing segmenation. For a particular organ of interest and a particular reference image, ten seed points could be found since there are 10 remaining images that can be used for testing. Each result entry in the table is the ratio of viable seed points $(0$ to 10) to the number of test volumes tried (which is equal to 10 here)

\begin{tabular}{c|ccccccc} 
Volume ID & Right Lung & Left Lung & Urinary Bladder & Right Kidney & Left Kidney & Liver & Spleen \\
\hline 100 & 1.0 & 1.0 & 1.0 & 1.0 & 0.1 & 1.0 & 0.1 \\
105 & 0.0 & 1.0 & 1.0 & 1.0 & 1.0 & 1.0 & 0.1 \\
106 & 1.0 & 1.0 & 1.0 & 1.0 & 0.1 & 1.0 & 0.0 \\
108 & 1.0 & 1.0 & 1.0 & 1.0 & 1.0 & 1.0 & 0.1 \\
109 & 1.0 & 1.0 & 0.0 & 1.0 & 1.0 & 1.0 & 0.1 \\
110 & 1.0 & 1.0 & 0.0 & 0.0 & 0.1 & 1.0 & 0.1 \\
112 & 1.0 & 1.0 & 1.0 & 1.0 & 0.0 & 1.0 & 1.0 \\
113 & 1.0 & 1.0 & 1.0 & 0.1 & 1.0 & 1.0 & 1.0 \\
127 & 1.0 & 1.0 & 1.0 & 1.0 & 0.1 & 0.6 & 0.1 \\
128 & 1.0 & 1.0 & 1.0 & 0.0 & 0.0 & 0.0 & 0.1 \\
131 & 1.0 & 1.0 & 1.0 & 1.0 & 0.8 & 1.0 & 0.1
\end{tabular}

Out of 770 calculated seed points, 557 were found to be viable. That translates to a success rate of $72.3 \%$.

\section{DISCUSSIONS AND FUTURE WORK}

This article presents a very simple, and easy-to-implement approach for finding seed points for segmentation and eventual identification of any organ based on annotated 3D training data. Obtained results indicate that it is indeed effective in finding seed points within the target organs and that a simple segmentation algorithm can subsequently delineate organs with a respectable degree of accuracy. It can be noted that the 3D probability maps themselves can be used as segmentations in areas where little contrast exists in the test volume itself. Moreover, the method is not restricted to CT volumes and can be used on any modality. In fact, the use of 
mutual information as a cost metric in the non-rigid registration algorithm allows the transfer of annotations from one modality to another modality with no great difficulty.

Moreover, in this paper, the volumes used for training and testing were manually separated in terms of patient body shape. As a reminder, the goal of doing that was to avoid a large mismatch between reference and training volumes, which would subsequently translate into lower registration error and better seed point viability. However, that can be avoided in the future by asking clinicians to tag new patients as underweight, normal, overweight, or obese during data acquisition. This relatively quick and painless solution to the error minimisation challenge would not be difficult to implement and would make our method more viable than when no such pre-processing is performed.

Owing to the currently limited size of the dataset used, a limited set of results were presented. Notably, experiments were carried out only for patients with a normal body shape. A more expansive set of results will be gathered as more data are acquired for testing the proposed method. In that way, it will become possible to repeat the experiments for all body shapes. It will also become possible to carry out a more comprehensive study of the success rate of finding seed points falling within the confines of the target organ. Testing will also be extended to MR images as well as full-body volumes. A large quantity of such images are already available in VISCERAL and even more datasets will become available with time through the MD-PAEDIGREE project ${ }^{\ddagger}$.

Finally, segmentation will fail if the computed seed point happens to lie outside the target organ in volumes under examination. Table 2 contains some instances of non-viable seed points that will lead to failed segmentations. Those occurred because the registration approach described in this paper was not always sufficiently accurate. Future work will seek to address that issue by either using a different registration approach or by optimising the registration parameters that were employed to obtain the reported results. Notwithstanding those difficulties, $72.3 \%$ of calculated seed points were found to be viable. The related publications mentioned in the introduction section had slightly different objectives such that, at the time of writing, our work cannot be directly compared with existing literature in the field.

\section{CONCLUSIONS}

Using our simple method, seed points for region-growing segmentation can be found in $72.3 \%$ of the cases where the reference and test volumes belong to patients with a normal body shape. Separating patient volumes according to body shapes has precluded situations where there is a significantly large mismatch in terms of body shape between the test and reference volumes, thereby leading to more accurate registration and better seed points.

It is clear that registration accuracy is the major tumbling block in the quest for better figures for the viability of seed points. In future work, the investment of some effort on our part in finding better registration algorithms than the one used in this paper will undoubtedly help us increase the success rate even further. In addition, with the expected availability of a larger quantity and variety of images for training and testing, it will become possible to test and optimise our algorithm for all body shapes.

Once all proposed improvements are implemented, it is expected that the proposed method will help save time and play a significant role in the improvement in the way the ever-increasing mass of collected medical data is processed and stored.

\section{ACKNOWLEDGMENTS}

This work was partially funded by the EU FP7 MD-Paedigree project (grant agreement 600932).

\footnotetext{
${ }^{\ddagger}$ MD-PAEDIGREE: http://www.md-paedigree.eu/ [Online; accessed 19-January-2015].
} 


\section{REFERENCES}

1. Haux, R., "Hospital information systems - past, present, future," International Journal of Medical Informatics 75, 268-281 (2005).

2. Müller, H., Zhou, X., Depeursinge, A., Pitkanen, M., Iavindrasana, J., and Geissbuhler, A., "Medical visual information retrieval: State of the art and challenges ahead," in [2007 IEEE International Conference on Multimedia and Expo], 683-686, IEEE (2007).

3. Depeursinge, A., Foncubierta-Rodríguez, A., Van De Ville, D., and Müller, H., "Three-dimensional solid texture analysis and retrieval in biomedical imaging: review and opportunities," Medical Image Analysis 18(1), 176-196 (2014).

4. Doi, K., "Computer-aided diagnosis in medical imaging: Historical review, current status and future potential," CMIG 31, 198-211 (mar 2007).

5. Singh, P. P. and Singh, J., "Automatic seed placement in region growing image segmentation," Journal of Engineering Computers $\&$ Applied Sciences 2(7), 55-58 (2013).

6. Xing, F., Asman, A. J., Prince, J. L., and Landman, B. A., "Finding seeds for segmentation using statistical fusion," in [SPIE Medical Imaging], 831430-831430, International Society for Optics and Photonics (2012).

7. Warfield, S. K., Zou, K. H., and Wells, W. M., "Validation of image segmentation by estimating rater bias and variance," Philosophical Transactions of the Royal Society A: Mathematical, Physical and Engineering Sciences 366(1874), 2361-2375 (2008).

8. Commowick, O. and Warfield, S. K., "A continuous staple for scalar, vector, and tensor images: an application to dti analysis," Medical Imaging, IEEE Transactions on 28(6), 838-846 (2009).

9. Xing, F., Soleimanifard, S., Prince, J. L., and Landman, B. A., "Statistical fusion of continuous labels: identification of cardiac landmarks," in [Proceedings-Society of Photo-Optical Instrumentation Engineers], 7962, NIH Public Access (2011).

10. Woolrich, M. W., Jbabdi, S., Patenaude, B., Chappell, M., Makni, S., Behrens, T., Beckmann, C., Jenkinson, M., and Smith, S. M., "Bayesian analysis of neuroimaging data in fsl," Neuroimage 45(1), S173-S186 (2009).

11. Klein, S., Staring, M., Murphy, K., Viergever, M. A., and Pluim, J. P., "Elastix: a toolbox for intensity-based medical image registration," IEEE Transactions on medical imaging 29(1), 196-205 (2010).

12. Mubarak, D. M. N., Sathik, M. M., Beevi, S. Z., and Revathy, K., "A hybrid region growing algorithm for medical image segmentation," International Journal of Computer Science E Information Technology (IJCSIT) Vol 4 (2012).

13. Harris, C. and Stephens, M., "A combined corner and edge detection," in [Proceedings of The Fourth Alvey Vision Conference], 147-151 (1988).

14. Langs, G., Müller, H., Menze, B. H., and Hanbury, A., "Visceral: Towards large data in medical imaging challenges and directions," Lecture Notes in Computer Science 7723, 92-98 (2013).

15. Fedorov, A., Beichel, R., Kalpathy-Cramer, J., Finet, J., Fillion-Robin, J.-C., Pujol, S., Bauer, C., Jennings, D., Fennessy, F., Sonka, M., et al., "3d slicer as an image computing platform for the quantitative imaging network," Magnetic Resonance Imaging (2012).

16. Berger, M., [Geometry. I. Universitext], Springer-Verlag, Berlin (1987).

17. Unser, M., Aldroubi, A., and Eden, M., "B-spline signal processing. i. theory," IEEE Transactions on Signal Processing 41(2), 821-833 (1993).

18. Unser, M., Aldroubi, A., and Eden, M., "B-spline signal processing. ii. efficiency design and applications," IEEE Transactions on Signal Processing 41(2), 834-848 (1993).

19. Unser, M., "Splines: A perfect fit for signal and image processing," IEEE Signal Processing Magazine 16(6), 22-38 (1999).

20. Cover, T. M. and Thomas, J. A., "Entropy, relative entropy and mutual information," Elements of Information Theory , 12-49 (1991).

21. Mattes, D., Haynor, D. R., Vesselle, H., Lewellen, T. K., and Eubank, W., "Nonrigid multimodality image registration," Medical Imaging 4322(1), 1609-1620 (2001).

22. Mattes, D., Haynor, D. R., Vesselle, H., Lewellen, T. K., and Eubank, W., "Pet-ct image registration in the chest using free-form deformations," IEEE Transactions on Medical Imaging 22(1), 120-128 (2003). 